\title{
THE HENSELIAN DEFECT FOR VALUED FUNCTION FIELDS
}

\author{
JACK OHM
}

(Communicated by Louis J. Ratliff, Jr.)

\begin{abstract}
The notion of defect for finite algebraic extensions of valued fields is classical and due to Ostrowski. Recently Matignon has generalized Ostrowski's definition to $r k 1$ (residually transcendental) valued function fields and used it to prove a very sharp version of the genus reduction inequality for 1-dim function fields. The further generalization of the notion of defect to valued function fields of arbitrary $r k$ is treated here.
\end{abstract}

Let $\left(K / K_{0}, v\right)$ be a valued function field of $\operatorname{dim} n$, i.e., $K / K_{0}$ is a finitely generated field extension of deg of transcendence $n$ and $v$ is a valuation of $K$. Let $V_{0} \subset V, k_{0} \subset k$, and $G_{0} \subset G$ be the respective valuation rings, residue fields, and value groups of the extension $K_{0} \subset K$; and let $*$ denote image under the $v$-residue map $V \rightarrow V / m_{V}=k$.

A transcendence basis $t=\left\{t_{1}, \ldots, t_{n}\right\}$ of $K / K_{0}$ will be called a residually transcendental (abbreviated tr.) basis of the valued function field if $v\left(t_{i}\right) \geq 0$ $(i=1, \ldots, n)$ and the set of $v$-residues $t^{*}=\left\{t_{1}^{*}, \ldots, t_{n}^{*}\right\}$ is algebraically independent over $k_{0}$. The function field will be called residually tr. if there exists a residually tr. basis.

A transcendence basis $t$ is residually tr. iff $v \mid K_{0}(t)$ is the inf extension, denoted $v_{0}^{t}$, of $v_{0}$ w.r.t. $t$, i.e., iff for all $f(t)$ in $K_{0}[t], v(f)=$ the inf of the values of the coefficients of $f$. Note that the value group of $v_{0}^{t}$ is clearly $G_{0}$, and the residue field is $k_{0}\left(t^{*}\right)$. (Cf. [3, p. 161, Proposition 2].)

If $t$ is a residually tr. basis, the henselian defect at $t$ is defined to be

$$
D^{h}(t):=\left[K^{h}: K_{0}(t)^{h}\right] / I R,
$$

where $K^{h}$ denotes henselization, $I=\left[G: G_{0}\right]$, and $R=\left[k: k_{0}\left(t^{*}\right)\right]$.

We shall prove here the

0 . Independence Theorem. Let $\left(K / K_{0}, v\right)$ be a residually tr. valued function field. Then $D^{h}(t)$ is independent of the choice of residually tr. basis $t$.

The case that $K / K_{0}$ is simple tr. has been proved in [13, Theorem 2.2]. Also, Matignon [10, p. 191, Corollary 1] has proved that if $\mathrm{rk} v=1$, then the

Received by the editors July 5,1988 .

1980 Mathematics Subject Classification (1985 Revision). Primary 13A18, 12 F20.

Key words and phrases. Valued function field, defect. 
completion defect $D^{-}(t)$ is independent of $t$, where $D^{-}(t)$ is defined analogously to $D^{h}(t)$ by using the completion instead of the henselization; this result, which plays a key role in the proof of Matignon's genus reduction inequality [10], follows readily from Theorem 0 ; see 4.2. Finally, we should mention that F.-V. Kuhlmann has independently proved the Independence Theorem; see 4.3.

\section{Preliminaries}

Fix throughout $\S 1$ a finite algebraic extension of valued fields $\left(L_{0}, w_{0}\right) \subset(L, w)$, with value groups $H_{0} \subset H$ and residue fields $1_{0} \subset 1$.

We use $L^{h}$ to denote henselization and $L^{-}$to denote completion. Recall that both $L^{h}$ and $L^{-}$have the same residue field and value group as $L$ and that any finite generating set for $L / L_{0}$ is also a generating set for $L^{h} / L_{0}^{h}$ and

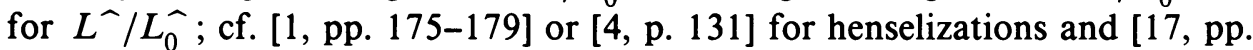
$45-47, \S \S 4$ and 5$]$ or $\left[3\right.$, p. 121] for completions. We shall call $\left[L^{h}: L_{0}^{h}\right]$ the henselian deg of $w / w_{0}$.

A set of extensions $w_{1}=w, w_{2}, \ldots, w_{n}$ of $w_{0}$ to $L$ is called a complete set of extensions of $w_{0}$ to $L$ if every extension of $w_{0}$ to $L$ is equivalent to one of these and no two of these are equivalent.

\subsection{The fundamental equality.}

$$
\left[L: L_{0}\right]=\sum_{i=1}^{n}\left[\left(L, w_{i}\right)^{h}:\left(L_{0}, w_{0}\right)^{h}\right]
$$

(Apply [4, p. 125, (17.3)] and the fact that, in the terminology of that reference, $L_{0}^{h} / L_{0}$ is a separable, "allowable" extension.) The sum is taken over a complete set of extensions $w_{1}=w, \cdots, w_{n}$ of $w_{0}$ to $L$. In words, the fundamental equality says that the deg is the sum of the Henselian degs.

1.2. Definition of the defects. Let $e=\left[H: H_{0}\right]$ and $f=\left[1: 1_{0}\right]$; that is, $e$ is the index and $f$ is the residue deg. We define two notions of defect for the extension $\left(L_{0}, w_{0}\right) \subset(L, w)$, the henselian defect and the completion defect, respectively, as follows:

(i) $\operatorname{def}^{h}\left(w / w_{0}\right)=\left[(L, w)^{h}:\left(L_{0}, w_{0}\right)^{h}\right] / e f$

(ii) $\operatorname{def}^{\sim}\left(w / w_{0}\right)=\left[(L, w)^{\wedge}:\left(L_{0}, w_{0}\right)^{\wedge}\right] / e f$.

We can now restate the fundamental equality 1.1 :

$$
\left[L: L_{0}\right]=\sum_{i=1}^{n} \operatorname{def}^{h}\left(w_{i} / w_{0}\right) e_{i} f_{i} .
$$

Note that each defect is a rational number $\geq 1$. Much of the usefulness of these notions is due to the classical

Theorem. Let $p=\operatorname{char} l_{0}$ if $\operatorname{char} l_{0}>0$ and $p=1$ if $\operatorname{char} l_{0}=0$.

(i) (Ostrowski) If rk $w_{0}=1$, then $\operatorname{def}^{\sim}\left(w / w_{0}\right)=p^{i}$ for some $i \geq 0$; and if $w_{0}$ is discrete rk 1 , then $\operatorname{def}^{\sim}\left(w / w_{0}\right)=1$ (cf. [16, p. 355] and [3, p. 148, Corollary 2]). 
(ii) (E. Artin-Ostrowski) If $\mathrm{rk} w_{0}$ is arbitrary, then $\operatorname{def}^{h}\left(w / w_{0}\right)=p^{i}$ for some $i \geq 0$ (cf. [1, p. 180, Proposition 15], or [3, p. 190, Exercise 9]).

\subsection{Comparison of the defects.}

Proposition ([13, §1.3]). $\operatorname{def}^{h}\left(w / w_{0}\right) \leq \operatorname{def}^{\wedge}\left(w / w_{0}\right) Q^{\wedge}\left(w / w_{0}\right)$, and $=$ holds if $\left(L_{0}, w_{0}\right)^{\wedge}$ is henselian (in particular, $=$ holds if $\left.\mathrm{rk} w_{0}=1\right)$. Here $Q^{-}\left(w / w_{0}\right)$ is called the inseparability quotient and is defined by

$$
Q^{\frown}\left(w / w_{0}\right)=\left[L: L_{0}\right]_{\text {ins }} /\left[(L, w)^{\uparrow}:\left(L_{0}, w_{0}\right)^{\curlywedge}\right]_{\text {ins }}
$$

where []$_{\text {ins }}$ denotes deg of inseparability.

Note that $Q^{-}\left(w / w_{0}\right)=1$ whenever $L_{0}^{\uparrow} / L_{0}$ or $L / L_{0}$ is separable, and in general equals $p^{i}$ for some $i \geq 0$, where $p=\operatorname{char} L_{0}$ (apply [19, p. 119, Corollary 2 and p. 114, Lemma 1]). For a discrete rk 1 example with $\left[L: L_{0}\right]=$ $Q^{-}\left(w / w_{0}\right)=p>0$, cf. [20, p. 62]; this example has $\operatorname{def}^{\sim}=1$ and $\operatorname{def}^{h}=p$. On the other hand, there exist examples with $\operatorname{def}^{h}=1$ and def $^{\wedge}$ an arbitrary rational number $\geq 1$; cf. [13, Example 2.5].

1.4 Stability (cf. [2, p. 160, Proposition 6], [7, p. 57]). The valued field $\left(L_{0}, w_{0}\right)$ is called stable if for every finite algebraic extension $(L, w)$ of $\left(L_{0}, w_{0}\right), \operatorname{def}^{h}\left(w / w_{0}\right)=1$.

\section{Proof of the theorem for $K_{0}$ Algebraically closed}

The terminology of the introduction will be in effect.

2.1. Theorem. Let $\left(K / K_{0}, v\right)$ be a residually $\operatorname{tr}$. valued function field, and let $t=\left\{t_{1}, \ldots, t_{n}\right\}$ be a residually $\operatorname{tr}$. basis. If $K_{0}$ is algebraically closed, then $\operatorname{def}^{h}\left(K / K_{0}(t)\right)=1$.

Proof. Let $v_{0}=v \mid K_{0}$ and $v_{0}^{t}=v \mid K_{0}(t)$. Note that $\mathrm{rk} v_{0}=\mathrm{rk} v_{0}^{t}=\mathrm{rk} v$.

Case (i). rk $v=1$. We work inside $K^{\curlywedge} . K_{0}$ is algebraically closed $\Rightarrow K_{0}^{\wedge}$ is algebraically closed $\left([2\right.$, p. 146 , Proposition 3$] \Rightarrow K_{0}^{\wedge}(t)$ is stable $([2$, p. 215 , Proposition 3]) $\Rightarrow \operatorname{def}^{h}\left(K_{0}^{\curlywedge} K / K_{0}^{\curlywedge}(t)\right)=1$, by 1.4 .

But by 1.3 , in rk 1

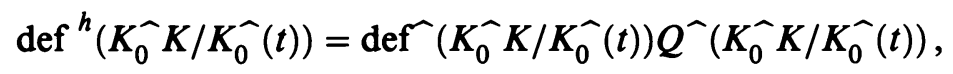

so $\operatorname{def}^{\wedge}\left(K_{0}^{\wedge} K / K_{0}^{\curlywedge}(t)\right)=1$ and $Q^{\wedge}\left(K_{0}^{\wedge} K / K_{0}^{\wedge}(t)\right)=1$.

Since $\left(K_{0}^{\wedge} K\right)^{\wedge}=K^{\wedge}$ and $\left(K_{0}^{\wedge}(t)\right)^{\wedge}=K_{0}(t)^{\wedge}$, we have by definition of $\operatorname{def}^{\wedge}, \operatorname{def}^{\wedge}\left(K / K_{0}(t)\right)=1$. It remains to show $Q^{\wedge}\left(K / K_{0}(t)\right)=1$, for then another application of 1.3 yields the desired result. Since we know $Q^{\wedge}\left(K_{0}^{\wedge} K /\right.$ $\left.K_{0}^{\hat{0}}(t)\right)=1$, it suffices to prove the

Claim. $\left[K: K_{0}(t)\right]_{\text {ins }}=\left[K_{0}^{\curlywedge} K: K_{0}^{\curlywedge}(t)\right]_{\text {ins }}$. Since $t$ is a residually tr. basis, the set $t$ remains algebraically independent over $K_{0}^{\hat{0}}$. Therefore $K$ and $K_{0}^{\wedge}$ are algebraically independent over $K_{0}$; and since $K_{0}$ is algebraically closed, then 
$K$ and $K_{0}^{-}$are linearly disjoint over $K_{0}$ (cf. [18, p. 18, Theorem 5]). The claim follows by [19, p. 114, Lemma 1]. Q.E.D. for Case (i).

Case (ii). $\mathrm{rk} v$ is finite. We proceed by induction on $\mathrm{rk} v$. We shall prove in Case (ii) the following equivalent form of Theorem 2.1.

$2.1^{\prime}$ Theorem. Let $K_{0}$ be an algebraically closed field, let $t$ be a finite set of indeterminates, and let $K$ be a finite algebraic extension of $K_{0}(t)$. Let $v_{0}$ be a valuation of $K_{0}, v_{0}^{t}$ be the inf extension of $v_{0}$ w.r.t. $t$, and $v_{1}, \ldots, v_{m}$ be a complete set of extensions of $v_{0}^{t}$ to $K$. Then

$$
\left[K: K_{0}(t)\right]=R\left(v_{1} / v_{0}^{t}\right)+\cdots+R\left(v_{m} / v_{0}^{t}\right),
$$

where $R\left(v_{i} / v_{0}^{t}\right)=$ residue deg of $v_{i} / v_{0}^{t}$.

Assume $\mathrm{rk} v_{0}$ is finite, $>1$.

For any finite rk valuation of a field, there is a unique (up to equivalence) rk 1 valuation of the same field whose valuation ring contains the valuation ring of the given valuation. Let $w_{0}, w_{1}, \ldots, w_{m}$ be the rk 1 valuations corresponding in this way to $v_{0}, v_{1}, \ldots, v_{m}$. Some of $w_{1}, \ldots, w_{m}$ may be equivalent (i.e., have the same valuation ring), so let us restrict ourselves to a complete subset of inequivalent valuations, say $w_{1}, \ldots, w_{s}$. If $w_{0}^{t}$ denotes the inf extension of $w_{0}$ w.r.t. $t$, it follows that $w_{0}^{t}$ is the rk 1 valuation corresponding to $v_{0}^{t}$.

The rk $1 w$-valuations satisfy the hypothesis of $2.1^{\prime}$, and therefore by the previously proved rk 1 case,

$$
\left[K: K_{0}(t)\right]=R\left(w_{1} / w_{0}^{t}\right)+\cdots+R\left(w_{s} / w_{0}^{t}\right),
$$

where $R()$ denotes residue deg.

Now let $w$ be any one of the valuations $w_{1}, \ldots, w_{s}$, and let, say, $v_{1}, \ldots, v_{q}$ be those elements of $\left\{v_{1}, \ldots, v_{m}\right\}$ whose valuation rings are contained in the valuation ring of $w$ (i.e., $v_{1}, \ldots, v_{q}$ are those elements of $\left\{v_{1}, \ldots, v_{m}\right\}$ which are in the dependence class determined by $w$ ). Let $l_{0}=$ residue field of $w_{0}$, $l_{w}=$ residue field of $w$; and denote image under the $w$-residue map by (a bar). Then $l_{w}$ is finite algebraic over $l_{0}(\bar{t})$ and the set $\bar{t}$ is algebraically independent over $l_{0}$. Thus, we have valuations $\bar{v}_{0}$ of $l_{0},\left(v_{0}^{t}\right)^{-}=\bar{v}_{0}^{\bar{t}}$ of $l_{0}(\bar{t})$, and $\bar{v}_{1}, \ldots, \bar{v}_{q}$ of $l_{w}$ (where a valuation $v$ corresponds to a valuation $v$ by applying the $w$ residue map to the valuation ring of $v$ ).

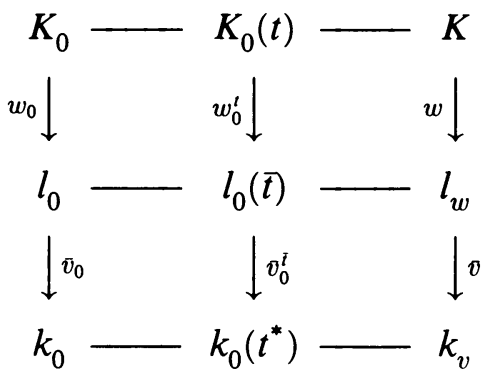


By induction hypothesis,

$$
R\left(w / w_{0}^{t}\right)=\left[l_{w}: l_{0}(\bar{t})\right]=R\left(\bar{v}_{1} / \bar{v}_{0}^{\bar{t}}\right)+\cdots+R\left(\bar{v}_{q} / \bar{v}_{0}^{\bar{t}}\right) .
$$

Since $R\left(v_{i} / v_{0}^{t}\right)=R\left(\bar{v}_{i} / \bar{v}_{0}^{\bar{t}}\right)$, we can substitute the expressions (2.4) into (2.3) to obtain the desired equality. Q.E.D. for Case (ii).

Case (iii). rk $v_{0}$ arbitrary. Our approach will be to drop down to a suitable extension $K_{0}^{\prime} \subset K^{\prime}$, where $K_{0}^{\prime}$ is the algebraic closure of a finitely generated extension of the prime field. This forces $\operatorname{rk}\left(v \mid K^{\prime}\right)$ to be finite, and we can then apply Case (ii).

First we need a lemma.

2.5 Lemma. Let $\left(K / K_{0}, v\right)$ be a residually tr. valued function field, let $t$ be a residually tr. basis, and let $K=K_{0}(t, a)$, where a denotes a finite generating set for $K / K_{0}(t)$.

Then there exists a finite subset $S$ of $K_{0}$ such that if $K_{0}^{\prime}$ is any subfield of $K_{0}$ containing $S$ and $K^{\prime}=K_{0}^{\prime}(t, a)$, then

(i) $K^{\prime} / K_{0}^{\prime}(t)$ is algebraic,

(ii) $\left[K^{h}: K_{0}(t)^{h}\right]=\left[K^{\prime h}: K_{0}^{\prime}(t)^{h}\right]$, and

(iii) residue deg $\left(K / K_{0}(t)\right) \leq$ residue deg $\left(K^{\prime} / K_{0}^{\prime}(t)\right)$.

Moreover, if $K_{0}^{\prime}$ is chosen to be algebraically closed, then = holds in (iii).

(For ease of notation, let $E^{h}, E^{\prime h}$ denote the left and right sides of (ii) and $R, R^{\prime}$ the left and right sides of (iii)).

Before proving 2.5, we shall finish the proof of Case (iii). Let $S$ be given by 2.5 ; let $K_{0}^{\prime}$ be the algebraic closure of $P(S)$, where $P$ is the prime subfield of $K_{0}$; and let $K^{\prime}=K_{0}^{\prime}(t, a)$, where $a$ is a generating set for $K / K_{0}(t)$, as in 2.5. Note that $v \mid K_{0}^{\prime}$ has finite rk (cf. [20, p. 8, Theorem 3]). Also, since $K_{0}^{\prime}$ and $K_{0}$ are algebraically closed, both $K / K_{0}(t)$ and $K^{\prime} / K_{0}^{\prime}(t)$ have index 1. By definition of $\operatorname{def}^{h}, \operatorname{Rdef}^{h}\left(K / K_{0}(t)\right)=E^{h}$ and $R^{\prime} \operatorname{def}^{h}\left(K^{\prime} / K_{0}^{\prime}(t)\right)=$ $E^{\prime h}$; by $2.5-$ (ii) $E^{h}=E^{\prime h}$; and by the final assertion of $2.5, R=R^{\prime}$. Thus, $\operatorname{def}^{h}\left(K / K_{0}(t)\right)=\operatorname{def}^{h}\left(K^{\prime} / K_{0}^{\prime}(t)\right)$. But by Case (ii) $\operatorname{def}^{h}\left(K^{\prime} / K_{0}^{\prime}(t)\right)=1$. This concludes the proof of 2.1 .

Proof of Lemma 2.5. Let $v_{0}=v \mid K_{0}$ and $v_{0}^{t}=v \mid K_{0}(t)$; let $v_{1}=v, \ldots, v_{m}$ be a complete set of extensions of $v_{0}^{t}$ to $K$; let $k_{i}=$ residue field of $v_{i}$ $(i=0, \ldots, m)$; and write $k_{i}=k_{0}\left(t^{*}, z^{(i)}\right)$, where $z^{(i)}$ denotes a finite set of elements and $t^{*}$ denotes the set of $v_{0}^{t}$-residues of the elements of $t$.

By enlarging the set $a$ if necessary, we may assume $a$ contains a set of elements having $v_{i}$-residue $z^{(i)}(i=1, \ldots, m)$.

Let $F$ be the finite subset of $K_{0}(t)$ consisting of the union of the following three sets:

$F_{1}$. Choose a finite basis in $K_{0}(t)[X]$ for the ideal of $a$ over $K_{0}(t)$, and let $F_{1}$ be the set of $K_{0}(t)$-coefficients appearing in the elements of this basis. 
$F_{2}$. For each $i$ in $\{1, \ldots, m\}$ choose a finite basis in $k_{0}\left(t^{*}\right)[X]$ for the ideal of $z^{(i)}$ over $k_{0}\left(t^{*}\right)$. For each $k_{0}\left(t^{*}\right)$-coefficient $c^{*}$ appearing in these basis elements select an element $c$ in $K_{0}(t)$ having $c^{*}$ as its $v_{0}^{t}$-residue, and let $F_{2}$ be the resulting set of $c$ 's.

$F_{3}$. For each $i \neq j$ in $\{1, \ldots, m\}$, choose an element $b_{i j}$ in $K=K_{0}(t)[a]$ such that $v_{i}\left(b_{i j}\right) \geq 0$ and $v_{j}\left(b_{i j}\right)<0$. Write each $b_{i j}$ as an element of $K_{0}(t)[a]$, and let $F_{3}$ be the set of $K_{0}(t)$-coefficients appearing in the resulting expressions for the $b_{i j}$.

Finally, write each element of $F$ as an element of $K_{0}(t)$, and let $S$ be the set of $K_{0}$-coefficients appearing in the resulting expressions.

Now let $K_{0}^{\prime}$ be any subfield of $K_{0}$ containing $S$; let $K^{\prime}=K_{0}^{\prime}(t, a)$; and let $v_{0}^{\prime}=v\left|K_{0}^{\prime}, v_{0}^{\prime t}=v\right| K_{0}^{\prime}(t)$, and $v_{i}^{\prime}=v_{i} \mid K^{\prime}(i=1, \ldots, m)$. Note that $F \subset$ $K_{0}^{\prime}(t)$; and, in particular, $F_{3} \subset K_{0}^{\prime}(t)$ implies that $v_{1}^{\prime}, \ldots, v_{m}^{\prime}$ are inequivalent, and $F_{1} \subset K_{0}^{\prime}(t)$ implies 2.5-(i) (cf. [18, p. 15, Theorem 3]).

Fix a $v_{i}$, and work inside a henselization $\left(K, v_{i}\right)^{h}$. Then $K^{h}=K_{0}(t)^{h}(a)$ and $K^{\prime h}=K_{0}^{\prime}(t)^{h}(a)$, so $K_{0}^{\prime}(t)^{h} \subset K_{0}(t)^{h}$ implies

$$
\left[K^{h}: K_{0}(t)^{h}\right] \leq\left[K^{\prime h}: K_{0}^{\prime}(t)^{h}\right] \text {. }
$$

For ease of notation, let $E_{i}^{h}$ and $E_{i}^{\prime h}$ denote the left and right sides of (2.6), so that (2.6) reads $E_{i}^{h} \leq E_{i}^{\prime h}$.

Since $F_{1} \subset K_{0}^{\prime}(t)$, we have (cf. [18, p. 15, Theorem 3]) $K_{0}(t)$ and $K_{0}^{\prime}(t)(a)$ are linearly disjoint over $K_{0}^{\prime}(t)$. Therefore

$$
\left[K_{0}(t)(a): K_{0}(t)\right]=\left[K_{0}^{\prime}(t)(a): K_{0}^{\prime}(t)\right],
$$

or, in abbreviated notation, $E=E^{\prime}$.

Putting together $1.1,(2.6)$, and (2.7), we have

$$
\begin{aligned}
& E=E_{1}^{h}+\cdots+E_{m}^{h} \\
& \| \wedge|\quad \wedge| \\
& \ddot{E}^{\prime}=\hat{E}_{1}^{\prime h}+\cdots+\hat{E}_{m}^{\prime h}+\cdots+E_{n}^{\prime h},
\end{aligned}
$$

where the $E_{i}^{h}$ (resp. the $E_{j}^{\prime h}$ ) are the henselian degrees of a complete set of extensions of $v_{0}^{t}$ (resp. $\left.v_{0}^{\prime t}\right)$ to $K$ (resp. $K^{\prime}$ ).

It follows from (2.8) that $m=n$ and that the inequalities of (2.8) are equalities. In particular, since $v=v_{1}$, the equality $E_{1}^{h}=E_{1}^{\prime h}$ yields 2.5 -(ii).

Now let $k_{i}^{\prime}=$ residue field of $v_{i}^{\prime}, i=0, \ldots, m$. Note that by our intial enlargement of $a, k_{0}^{\prime}\left(t^{*}, z^{(i)}\right) \subset k_{i}^{\prime}(i=1, \ldots, m)$; and therefore

$$
\left[k_{i}^{\prime}: k_{0}^{\prime}\left(t^{*}\right)\right] \geq\left[k_{0}^{\prime}\left(t^{*}\right)\left(z^{(i)}\right): k_{0}^{\prime}\left(t^{*}\right)\right] \quad(i=1, \ldots, m) .
$$

Since $F_{2} \subset K_{0}^{\prime}(t)$, we have (cf. [18, p. 15, Theorem 3]) $k_{0}\left(t^{*}\right)$ and $k_{0}^{\prime}\left(t^{*}\right)\left(z^{(i)}\right)$ are linearly disjoint over $k_{0}^{\prime}\left(t^{*}\right)$; and therefore

$$
\left[k_{0}^{\prime}\left(t^{*}\right)\left(z^{(i)}\right): k_{0}^{\prime}\left(t^{*}\right)\right]=\left[k_{0}\left(t^{*}\right)\left(z^{(i)}\right): k_{0}\left(t^{*}\right)\right] \quad(i=1, \ldots, m) .
$$


Then (2.9) and (2.10) yield 2.5-(iii).

Finally, assume $K_{0}^{\prime}$ is algebraically closed. Then $k_{0}^{\prime}$ is also algebraically closed, and therefore by $[9$, p. 58, Theorem 4$], k_{0}^{\prime}\left(t^{*}, z^{(i)}\right)$ is algebraically closed in $k_{0}\left(t^{*}, z^{(i)}\right)$. But

$$
k_{0}^{\prime}\left(t^{*}, z^{(i)}\right) \subset k_{i}^{\prime} \subset k_{0}\left(t^{*}, z^{(i)}\right)
$$

and $k_{i}^{\prime}$ is algebraic over $k_{0}^{\prime}\left(t^{*}, z^{(i)}\right)$; so then $k_{0}^{\prime}\left(t^{*}, z^{(i)}\right)=k_{i}^{\prime}$. Therefore (2.10) yields the final assertion of the lemma.

\section{Proof of THE THEOREM}

3.1 Theorem (Independence of $\operatorname{def}^{h}$ for residually tr. function fields). Let $\left(K / K_{0}, v\right)$ be a residually tr. valued function field. Then for any two residually tr. bases $t^{(1)}, t^{(2)}$ of the function field, $\operatorname{def}^{h}\left(K / K_{0}\left(t^{(1)}\right)\right)=\operatorname{def}^{h}\left(K / K_{0}\left(t^{(2)}\right)\right)$.

First we need an addition to Lemma 2.5 .

2.5' Lemma. Assume the hypotheses and notation of 2.5. If $K_{0}$ is algebraically closed, then

(iv) index $\left(K^{\prime} / K_{0}^{\prime}(t)\right)=\operatorname{def}^{h}\left(K^{\prime} / K_{0}^{\prime}(t)\right)=1$.

Proof. Let $I^{\prime}=\operatorname{index}\left(K^{\prime} / K_{0}^{\prime}(t)\right)$. By 2.5-(ii) and (iii) and the definition of $\operatorname{def}^{h}$, we have (in the notation of 2.5)

$$
\operatorname{Rdef}^{h}\left(K / K_{0}(t)\right)=E^{h}=E^{\prime h}=\mathrm{I}^{\prime} \mathrm{R}^{\prime} \operatorname{def}{ }^{h}\left(K^{\prime} / K_{0}^{\prime}(t)\right) \geq R^{\prime} \geq R .
$$

But $\operatorname{def}^{h}\left(K / K_{0}(t)\right)=1$ by 2.1 ; so the inequalities of (3.2) must actually be equalities. Then $\mathrm{I}^{\prime} \mathrm{R}^{\prime} \operatorname{def}^{h}\left(K^{\prime} / K_{0}^{\prime}(t)\right)=R^{\prime}$, which implies $\mathrm{I}^{\prime} \operatorname{def}^{h}\left(K^{\prime} / K_{0}^{\prime}(t)\right)=$ 1 .

3.3 Proof of 3.1. Let $K_{0}^{\mathrm{alg}}$ be the algebraic closure of $K_{0}$, and fix an extension (again denoted $v$ ) of $v$ to $K_{0}^{\text {alg }} K$. By $2.5^{\prime}$ applied to $K_{0}^{\text {alg }} \subset K_{0}^{\text {alg }} K$, there exists a finite algebraic extension $K_{0}^{\prime}$ of $K_{0}$ such that $\operatorname{def}^{h}\left(K^{\prime} / K_{0}^{\prime}\left(t^{(1)}\right)\right)=$ $\operatorname{def}^{h}\left(K^{\prime} / K_{0}^{\prime}\left(t^{(2)}\right)\right)=1$, where $K^{\prime}=K_{0}^{\prime} K$.

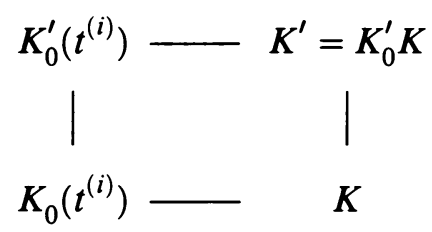

Since $\operatorname{def}^{h}\left(K^{\prime} / K_{0}^{\prime}\left(t^{(i)}\right)\right) \operatorname{def}^{h}\left(K_{0}^{\prime}\left(t^{(i)}\right) / K_{0}\left(t^{(i)}\right)\right)=\operatorname{def}^{h}\left(K^{\prime} / K\right) \operatorname{def}^{h}\left(K / K_{0}\left(t^{(i)}\right)\right)$, it only remains to note that $\operatorname{def}^{h}\left(K_{0}^{\prime}\left(t^{(1)}\right) / K_{0}\left(t^{(1)}\right)\right)=\operatorname{def}^{h}\left(K_{0}^{\prime}\left(t^{(2)}\right) / K_{0}\left(t^{(2)}\right)\right)$, which is a consequence of the observation that the extensions $K_{0}\left(t^{(1)}\right) \subset K_{0}^{\prime}\left(t^{(1)}\right)$ and $K_{0}\left(t^{(2)}\right) \subset K_{0}^{\prime}\left(t^{(2)}\right)$ are isomorphic as valued field extensions. 


\section{REMARKS}

4.1. Let $\left(K_{0}, v_{0}\right)$ be a valued field, and let $v_{0}^{t}$ be the inf extension of $v_{0}$ w.r.t. a set of $n$ indeterminates $t=\left\{t_{1}, \ldots, t_{n}\right\}$.

Theorem 2.1 can be rephrased as follows:

Theorem. If $K_{0}$ is algebraically closed, then $\left(K_{0}(t), v_{0}^{t}\right)$ is stable.

In the same vein, the argument of 3.3 yields the more general

Theorem. If $\left(K_{0}, v_{0}\right)$ is stable, then $\left(K_{0}(t), v_{0}^{t}\right)$ is stable.

Proof. Proceed as in 3.3, except that to see $\operatorname{def}^{h}\left(K_{0}^{\prime}(t) / K_{0}(t)\right)=1$ in 3.3, use the

Lemma. Let $\left(K_{0}, v_{0}\right) \subset\left(K_{1}, v_{1}\right)$ be an extension of valued fields with $K_{1} / K_{0}$ finite algebraic; let $t=\left\{t_{1}, \ldots, t_{n}\right\}$ be a set of $n$ indeterminates; and let $v_{0}^{t}, v_{1}^{t}$ be the inf extensions of $v_{0}, v_{1}$ resp. w.r.t. $t$. Then $\operatorname{def}^{h}\left(v_{1} / v_{0}\right)=\operatorname{def}^{h}\left(v_{1}^{t} / v_{0}^{t}\right)$. Proof (Matignon). Since $v_{1} / v_{0}$ and $v_{1}^{t} / v_{0}^{t}$ have the same index and residue $\mathrm{deg}$, the desired equality is equivalent to $\left[\left(K_{1}, v_{1}\right)^{h}:\left(K_{0}, v_{0}\right)^{h}\right]=$ $\left[\left(K_{1}(t), v_{1}^{t}\right)^{h}:\left(K_{0}(t), v_{0}^{t}\right)^{h}\right]$.

Let $v_{1}, v_{2}, \ldots, v_{n}$ be a complete set of extensions of $v_{0}$ to $K_{1}$. Then the corresponding inf extensions $v_{1}^{t}, v_{2}^{t}, \ldots, v_{n}^{t}$ form a complete set of extensions of $v_{0}^{t}$ to $K_{1}(t)$. Therefore, by 1.1 ,

$$
\begin{gathered}
{\left[K_{1}: K_{0}\right]=\sum_{i=1}^{n}\left[\left(K_{1}, v_{i}\right)^{h}:\left(K_{0}, v_{0}\right)^{h}\right]} \\
\| \\
{\left[K_{1}(t): K_{0}(t)\right]=\sum_{i=1}^{n}\left[\left(K_{1}(t), v_{i}^{t}\right)^{h}:\left(K_{0}(t), v_{0}^{t}\right)^{h}\right] .}
\end{gathered}
$$

But $\left[\left(K_{1}, v_{i}\right)^{h}:\left(K_{0}, v_{0}\right)^{h}\right] \geq\left[\left(K_{1}(t), v_{i}^{t}\right)^{h}:\left(K_{0}(t), v_{0}^{t}\right)^{h}\right] \quad(i=1, \ldots, n)$, since any finite generating set for $K_{1} / K_{0}$ is also a generating set for $K_{1}^{h} / K_{0}^{h}$ and $K_{1}(t)^{h} / K_{0}(t)^{h}$; so it follows that these inequalities must actually be equalities.

The rk 1 case of the first theorem is due to Grauert-Remmert [5, p. 119] and that of the second theorem to Gruson [7, p. 66, Theorem 3]; cf. [2, pp. 214-220] for "a simplified version of Gruson's approach."

4.2. Matignon [10, p. 191, Corollary 1] has proved that $\operatorname{def}^{\sim}\left(K / K_{0}(t)\right)$ is independent of $t$ in the rk 1 case. (A proof of this result in the simple tr. case is given in [12, Theorem 2.5]. Note also that the rk 1 hypothesis is necessary by [12, Example 2.6].) This result is equivalent to the rk 1 case of our independence theorem; for in rk 1 , by 1.3 ,

$$
\operatorname{def}^{h}\left(K / K_{0}(t)\right)=\operatorname{def}^{\Upsilon}\left(K / K_{0}(t)\right) Q^{\frown}\left(K / K_{0}(t)\right),
$$


and by [13, Remark 2.4.6] $Q^{-}\left(K / K_{0}(t)\right)$ is independent of $t$ (for any residually tr. valued function field).

4.3. Recent interest in the notion of defect has been stimulated by the key role it plays in the proof of Matignon's remarkable genus reduction inequality [10] (for 1 -dim rk 1 valued function fields), and by efforts to generalize this result to valuations of arbitrary rk; cf. [6], [8], [11]. In addition, in [12] and [13] the defect supplied the missing ingredient needed for the proof of the conjectures of [14] and [15] concerning the structure of simple tr. extensions of valued fields.

The proof of the independence theorem given here was inspired, in bare outline, by the proof of the simple tr . case given in [13, Theorem 2.2-(i)] and [12, Theorem 2.5]. The technique of reducing to rk 1 is classical, but it was brought to my attention by a letter (October 1987) from Barry Green and F. Pop to Matignon, in which they pointed out how it could be used to remove the rk 1 restriction from another of Matignon's results. Recently it was also brought to my attention that F.-V. Kuhlmann of Heidelberg had already used and proved generalizations of the principal results given here in his work towards his thesis.

\section{REFERENCES}

1. J. Ax, A metamathematical approach to some problems in number theory, 1969 Number Theory Institute, Proc. Sympos. Pure Math., vol. XX, Amer. Math. Soc., Providence, R.I., 1971, pp. 161-194.

2. S. Bosch, U. Güntzer, and R. Remmert, Non-archimedean analysis, Grundlehren Math. Wiss., no. 261, Springer-Verlag, Berlin, 1984.

3. N. Bourbaki, Algèbre commutative, Chaps. 5 and 6, Act. Sci. et Indust. 1308, Hermann, Paris, 1964.

4. O. Endler, Valuation theory, Springer-Verlag, New York, 1972.

5. H. Grauert and R. Remmert, Über die Methode der diskret bewerteten Ringe in der nichtarchimedischen Analysis, Invent. Math. 2 (1966), 87-133.

6. B. Green and F. Pop, Remarks on good reduction in valued function fields, preliminary manuscript, Heidelberg, January 1988.

7. L. Gruson, Fibrés vectoriels sur un polydisque ultramétrique, Ann. Sci. Ecole Norm. Sup. 1 (1968), 45-89.

8. F.-V. Kuhlmann, Ordinary defect, Matignon's defect and other notions of a defect for finite extensions of valued fields and a special class of valued algebraic function fields, preliminary manuscript, Heidelberg, April 22, 1988.

9. S. Lang, Introduction to algebraic geometry, Interscience Tracts No. 5, Interscience, New York, 1958.

10. M. Matignon, Genre et genre résiduel des corps de fonctions valués, Manuscripta Math. 58 (1987), 179-214.

11. __ Genre et genre résiduel des corps de fonctions valués (pour des valuations de rk arbitraire), preliminary manuscript, Bordeaux, May 3, 1988.

12. M. Matignon and J. Ohm, A structure theorem for simple transcendental extensions of valued fields, Proc. Amer. Math. Soc. , 104 (1988), 392-402.

13. _ Simple transcendental extensions of valued fields, III. The uniqueness property J. Math. Kyoto Univ. (to appear). 
14. J. Ohm, Simple transcendental extensions of valued fields, J. Math, Kyoto Univ. 22 (1982), 201-221.

15. __ Simple transcendental extensions of valued fields, II. A fundamental inequality, J. Math. Kyoto Univ. 25 (1985), 583-596.

16. A. Ostrowski, Untersuchungen zur arithmetischen Theorie der Körper, Math. Z. 39 (1935), 269-404.

17. P. Roquette, On the prolongation of valuations, Trans. Amer. Math. Soc. 88 (1958), 42-56.

18. A. Weil, Foundations of algebraic geometry, Amer. Math. Soc. Colloq. Publ, Vol. XXIX, Amer. Math. Soc., Providence, R.I., 1962.

19. O. Zariski and P. Samuel, Commutative algebra, Vol. I, Van Nostrand, Princeton, N.J., 1958.

20 _, Commutative algebra, Vol. II, Van Nostrand, Princeton, N.J., 1960.

Department of Mathematics, Louisiana State University, Baton Rouge, Louisiana 70803 